\title{
ESTUDO TEÓRICO DAS TRANSIÇÕES ELETRÔNICAS USANDO MÉTODOS SIMPLES E SOFISTICADOS
}

\author{
Nelson H. Morgon \\ Instituto de Química, Universidade Estadual de Campinas, CP 6154, 13084-862 Campinas - SP, Brasil
}

Recebido em 20/6/12; aceito em 10/10/12; publicado na web em 18/2/13

\begin{abstract}
THEORETICAL STUDY OF ELECTRONIC TRANSITIONS USING SIMPLE AND SOPHISTICATED METHODS. In this paper, the use of both simple and sophisticated models in the study of electronic transitions was explored for a set of molecular systems: $\mathrm{C}_{2} \mathrm{H}_{4}, \mathrm{C}_{4} \mathrm{H}_{4}, \mathrm{C}_{4} \mathrm{H}_{6}, \mathrm{C}_{6} \mathrm{H}_{6}, \mathrm{C}_{6} \mathrm{H}_{8}$, " $\mathrm{C}_{8}$ ", $\mathrm{C}_{60}$, and $\left[\mathrm{H}_{2} \mathrm{NCHCH}(\mathrm{CHCH})_{k} \mathrm{CHNH}_{2}\right]^{+}$, where $\mathrm{k}=0$ to 4 . The simple model of the free particle (1D, 2D, and $3 \mathrm{D}$ boxes, rings or spherical surfaces), considering the boundary conditions, was found to yield similar results to the sophisticated theoretical methods such as EOM-CCSD/6-311++G** or TD(NStates=5,Root=1)-M06-2X/6-311++G**.
\end{abstract}

Keywords: free particle models; theoretical calculations; electronic transitions.

\section{INTRODUÇÃO}

O modo mais simples de se estudar espectros eletrônicos é utilizando-se o modelo da partícula (elétron) "livre"confinada, sujeita a determinadas condições de contorno e potencial nulo. A transição eletrônica é descrita pela diferença entre os valores das energias quantizadas de dois estados quaisquer. Deste modo, este modelo (na caixa, anel ou superfície esférica) pode ser de grande ajuda na compreensão inicial de conceitos de transições eletrônicas em moléculas que contêm elétrons delocalizados, por exemplo os presentes em ligações $\pi$, bem como na interpretação de seus espectros eletrônicos. ${ }^{1,2}$ É um modelo que aparece em muitos cursos de química quântica, em livros textos e artigos em periódicos de educação. Numerosos exemplos do modelo têm sido explorados, em uma, duas ou três dimensões ou em outras variações mais interessantes como "a partícula em uma garrafa de champagne" e a "partícula em uma caixa de triângulo equilátero". ${ }^{3}$

A partícula na caixa é o sistema mecânico quântico mais simples onde a Equação de Schroedinger pode ser resolvida exatamente. Podendo-se assim, utilizá-lo no ensinamento de conceitos fundamentais da Mecânica Quântica. O comportamento do elétron livre, em movimento sem restrições, pode ser aproximado pela descrição da mecânica clássica, e sua energia expressa em função de sua massa de repouso $\left(m_{0}\right)$ e de sua velocidade $(v)$, dada apenas pela componente cinética. No entanto, existem outras situações diversas, onde o elétron se comporta de modo "não convencional" como, por exemplo, quando ele está sujeito a um campo, sob a ação de um poço de potencial (modelo da partícula confinada), em ambientes atômico ou molecular, etc. Assumindo então o comportamento mecânico-quântico (caracterizado como onda ou como partícula). Se ele apresentar $v \approx c$ deve-se introduzir componentes relativísticas para uma descrição mais precisa. ${ }^{4}$

Há outras vantagens no uso do modelo de partícula da caixa, como na exemplificação de separação de variáveis (no caso de partículas em 2 ou 3 dimensões), ou no estudo do efeito de perturbação. ${ }^{5} \mathrm{O}$ modelo, com ou sem potencial é um excelente laboratório para ensinar e explorar detalhes adicionais do método variacional linear usando-se conjuntos de base não ortogonais. ${ }^{6}$ Já o modelo da partícula no anel pode ser usado como uma boa aproximação no estudo das transições eletrônicas em compostos aromáticos, como no estudo do azuleno. ${ }^{7}$

Em cursos de termodinâmica estatística é possível explorar aspectos de partículas não interagentes na discussão do princípio de correspondência e condensação de Bose-Einstein. A importância desta

e-mail: morgon@iqm.unicamp.br discussão é introduzir, num estágio preliminar de cursos de química quântica, conceitos sobre o comportamento de bósons e férmions. Demonstra-se que férmions idênticos tentam evitar-se uns aos outros e este efeito surge, não da repulsão Coulômbica, mas das restrições de simetria impostas à função de onda. ${ }^{3}$ É também útil em estudos das distribuições de momento da partícula na caixa. ${ }^{8}$

Além das aplicações didáticas, o uso do modelo tem sido utilizado na interpretação dos resultados de medições do transporte de hélio através de nanotubos semicondutores de carbono, obtidas por microscopia de força atômica; ${ }^{9}$ no estudo do movimento translacional de moléculas de $\mathrm{H}_{2} \mathrm{O}$ na cavidade de $\mathrm{Be}_{2} \mathrm{Al}_{3} \mathrm{Si}_{6} \mathrm{O}_{18} \mathrm{H}_{2} \mathrm{O} ;{ }^{10}$ no exame das medidas de absorção transientes da isomerização cis-trans pelo pigmento visual rodopsina presente na retina; ${ }^{11}$ na modelagem dos níveis de energia em sistemas descritos por quantum dots (porção da matéria caracterizada por semicondutância). Neste caso, o modelo da partícula é bastante adequado, pois procura descrever os excitons (estados ligados entre o elétron e a vacância) confinados nas três dimensões espaciais. $\mathrm{O}$ conceito de quantum dots tem sido relevante no estudo de sistemas com aplicações em transistores, células solares, LEDs, lasers de diodo e com perspectiva como qubits em computação quântica. ${ }^{12-17}$

\section{ASPECTOS TEÓRICOS}

\section{Do modelo da partícula na caixa}

O modelo da partícula (elétron de massa $m_{e}$ ) confinada, quer em sistemas definidos por caixa em uma, duas ou três dimensões (referenciadas por 1D, 2D ou 3D, respectivamente), ou em outros ambientes como anel ou superfície esférica, é útil no entendimento da resolução da Equação de Schroedinger possibilitando a obtenção do valor de energia $E$, como mostra a Equação 1.

$$
\hat{H} \Psi=E \Psi
$$

O operador Hamiltoniano, em coordenadas cartesianas $(x, y, z)$, que atua na partícula sujeita ao potencial nulo $V(\vec{r})$ e descrita pela função de onda ( $\Psi)$, é dado pela Equação 2,

$$
\hat{H}=-\frac{h^{2}}{8 \pi^{2} m_{e}}\left(\frac{\partial^{2}}{\partial x^{2}}+\frac{\partial^{2}}{\partial y^{2}}+\frac{\partial^{2}}{\partial z^{2}}\right)
$$

ou pela Equação 3, se este for representado em termos de coordenadas polares $(r, \theta, \phi)$ 
$\hat{H}=-\frac{h^{2}}{8 \pi^{2} m_{e}}\left[\frac{1}{r^{2}} \frac{\partial}{\partial r}\left(r^{2} \frac{\partial}{\partial r}\right)+\frac{1}{r^{2} \operatorname{sen} \theta} \frac{\partial}{\partial \theta}\left(\frac{\partial}{\partial \theta}\right)+\frac{1}{r^{2} \operatorname{sen}^{2} \theta} \frac{\partial}{\partial \phi^{2}}\right]$

No caso da partícula na caixa unidimensional de comprimento $a$, assumindo-se o potencial zero dentro da caixa e infinito fora, observando-se as condições de contorno para a função de onda $\Psi(0)=\Psi(a)=0$, obtém-se as autofunções normalizadas $\Psi_{\mathrm{n}}(\mathrm{x})$ e os autovalores $E_{n}$, Equações 4 e 5 , respectivamente

$$
\Psi_{n}(x)=\sqrt{\frac{2}{a}} \operatorname{sen}\left(\frac{n \pi x}{a}\right)
$$

$\mathrm{e}$

$$
E_{n}=\frac{h^{2}}{8 \mathrm{~m}_{e} a^{2}} n^{2}, \quad \mathrm{n}=1,2,3,4, \ldots
$$

As descrições para a partícula nas caixas em 2D e 3D podem ser feitas de modo análogo à anterior em 1D. O resultado final em termos das autofunções normalizadas e autovalores fica dependente das variáveis $(x, y, z)$, bem como das dimensões das caixas $(a, b, c)$, respectivamente.

No caso de duas dimensões é possível também a aplicação considerando-se o deslocamento da partícula em um anel de raio $r$ e comprimento $C$. Para isto a representação bidimensional pode ser feita em termos de coordenadas polares, utilizando-se apenas as coordenadas ( $r$ e $\phi)$ e mantendo-se $\theta$ fixo.

Se a resolução da equação de autovalores (Equação 1) for feita em coordenadas cartesianas, ${ }^{18}$ considerando-se as condições de contorno:

$$
\Psi(x)=\Psi(x+C)
$$

e

$$
\left(\frac{\partial \Psi}{\partial x}\right)_{x=0}=\left(\frac{\partial \Psi}{\partial x}\right)_{x=C}
$$

os resultados dos autovalores e autofunções correspondem a:

$$
E_{n}=\frac{h^{2}}{8 \pi^{2} m_{e} r^{2}} n^{2}
$$

$\mathrm{e}$

$$
\Psi_{n}(x)=\sqrt{\frac{2}{C}} \operatorname{sen}\left(\frac{2 n \pi x}{C}+a\right)
$$

onde $\alpha$ pode ter qualquer valor. Se a mesma resolução for feita em coordenadas polares, para um dado valor de $r$ e $\operatorname{com} \theta$ constante e igual a $\pi / 2$ o operador descrito na Equação 3 reduz-se a:

$$
\hat{H}=-\frac{h^{2}}{8 \pi^{2} m_{e}} \frac{1}{r^{2} \operatorname{sen}^{2} \theta} \frac{\partial^{2}}{\partial \phi^{2}}
$$

e sob as condição de contorno $\Psi(\phi)=\Psi(\phi+2 \pi)$ para a função de onda, a solução da Equação 1 fornece a mesma solução para a energia (Equação 8) e conjunto de autofunções

$$
\Psi_{n}(\phi)=\frac{1}{\sqrt{2 \pi r}} e^{ \pm i n \phi}
$$

O caso de uma partícula em um anel unidimensional é um exemplo instrutivo para o estudo da quantização do momento angular, ou seja, um elétron orbitando o núcleo. As funções de onda azimutais neste caso são idênticas às autofunções para a energia da partícula em um anel.

A delocalização eletrônica dos orbitais $\pi$ triplamente degenerados pode ser associada à forma de um anel, como mostra a Figura 1a.
Assim, este modelo simples também pode ser usado no estudo das transições eletrônicas para este sistema molecular e análogos. ${ }^{7}$

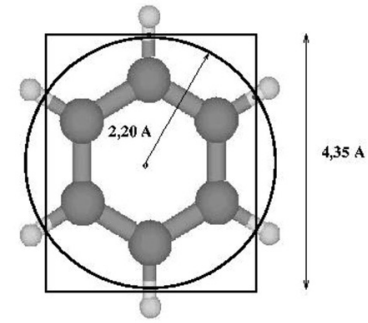

(a)

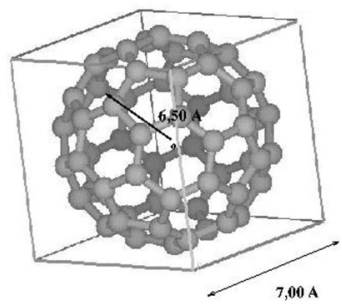

(b)
Figura 1. Representação de sistemas modelos para a delocalização eletrônica: (a) caixa $2 D$ e anel e (b) caixa $3 D$ e superfície esférica

O modelo da partícula na caixa em 3D é útil na compreensão do problema tridimensional em mecânica quântica, do efeito de degenerescência e, principalmente, na abordagem do conceito de separação de variáveis.

$$
\Psi(x, y, z)=X(x) Y(y) Z(z)
$$

Tal conceito é utilizado por exemplo, na representação da função de onda multieletrônica como um produtório de funções monoeletrônicas:

$$
\Psi\left(e_{1}, e_{2}, e_{3}, \ldots, e_{n}\right)=\prod_{i=1}^{n} \psi\left(e_{i}\right)
$$

Se o sistema for tratado em uma superfície esférica de raio $r$, a solução da equação de autovalor é apropriadamente feita em coordenadas polares e tendo as mesmas as soluções do modelo do rotor rígido, que são:

$$
\Psi(\theta, \phi)=Y_{1,1 \pm m}(\theta, \phi)
$$

onde são os harmônicos esféricos e as energias dos estados eletrônicos como:

$$
E=\frac{h^{2}}{8 \pi^{2} m_{e} r^{2}} n(n+1)
$$

Independentemente do sistema considerado, o cálculo do comprimento de onda $(\lambda)$ associado às transições eletrônicas entre estados de energias $E_{n}$, é dado pela expressão de Planck

$$
\Delta E=\left(E_{n f}-E_{n i}\right)=h v=\frac{h c}{\lambda}
$$

onde $E_{n i}$ e $E_{n f}$ são as energias eletrônicas dos estados inicial e final, respectivamente.

\section{Do modelo de estrutura eletrônica}

Considerando-se que a Equação de Schroedinger (Equação 1) não possui solução analítica para sistemas multieletrônicos, existem vários métodos aproximados que são utilizados no estudo da estrutura eletrônica de sistemas atômicos e moleculares. Tais aproximações ocorrem basicamente em dois grupos de teorias, aquelas baseadas no modelo de partículas independentes descritas a partir do método Hartree-Fock (HF) e as fundamentadas a partir da função de densidade eletrônica, os métodos da Teoria do Funcional de Densidade (do inglês DFT). ${ }^{19,20}$

Uma vez que a função de onda multieletrônica é desconhecida 
ela pode ser representada por expansões de funções monoeletrônicas como, por exemplo, orbitais atômicos ou moleculares para átomos ou moléculas, respectivamente. A representação destas funções pelos orbitais, do ponto de vista de facilitação da resolução de integrais eletrônicas e nucleares, bem como da implementação computacional, é demasiadamente vantajosa se for considerado o uso de conjuntos de funções base do tipo gaussianas, cujo núcleo é $e^{-\alpha \vec{r}}$, onde $\alpha$ representa expoentes das funções de base. As bases de Pople, do tipo 6-31G são bastante populares e facilmente implementáveis em muitos códigos computacionais. Além do que, possuem flexibilidade para a adição de funções extras denominadas difusas $(+)$ e de polarização $(*)$.

Por ser o método Hartree-Fock um modelo aproximado, pelo neglicenciamento, entre outros efeitos, da correlação eletrônica, existe atualmente uma grande diversidade de métodos que recuperam parte desta aproximação introduzindo-se então tal efeito. O método de Coupled Cluster (CC) é um dos mais rigorosos e tem atraído a atenção da comunidade científica. Neste método a função de onda é escrita numa expansão exponencial:

$$
\Psi=e^{\hat{T}}\left|\Phi_{0}\right|
$$

onde $\Phi_{0}$ são os orbitais de Hartree-Fock representados pelo determinante de Slater e $\hat{T}$ os operadores hermitianos de excitação que operam neste determinante. Estes operadores podem representar processos de excitações simples (S), duplas (D), triplas (T) etc. O número de termos na expansão acima, para processos envolvendo excitações de ordem maior (a partir de triplas), acarreta um aumento significativo do tempo de processamento computacional, inviabilizando o estudo de sistemas moleculares maiores. Existem alternativas nestes casos como, por exemplo, o uso de estimativas do efeito de excitações através de teorias "mais simples", como a teoria de perturbação. No caso de CCSD(T) tem-se o método de Coupled Cluster incluindo-se excitações simples e duplas de modo completo e as triplas de modo não explícito ou não iterativo, estimado seu efeito por perturbação.

A DFT é atualmente uma das ferramentas no estudo de estrutura eletrônica da matéria mais promissora e de maior sucesso. Sua aplicabilidade é possível para uma grande faixa de sistemas (de átomos, moléculas a sólidos), principalmente por prever uma grande variedade de propriedades moleculares: estrutura, frequências vibracionais, energias diversas, caminhos de reação, além de propriedades elétricas e magnéticas, entre outras. Ela fundamenta-se na obtenção de propriedades do sistema multieletrônico utilizando-se como funcional a densidade eletrônica $\rho(\vec{r})$. Sua base teórica é dada considerando-se dois teoremas fundamentais. ${ }^{21,22} \mathrm{O}$ primeiro teorema estabelece a determinação das propriedades do estado fundamental de sistemas multieletrônicos unicamentente em termos de $\rho(\vec{r})$ e o segundo que define um funcional de energia, $E[\rho(\vec{r})]$, para o sistema. $\mathrm{O}$ valor mínimo da energia do estado fundamental ocorre quando se tem a densidade eletrônica exata deste estado. De modo geral, a expressão para $E[\rho(\vec{r})]$, considerando-se um sistema de partículas não interagentes é:

$E[\rho]=T[\rho]-Z \int \frac{\rho(\vec{r})}{r} d \vec{r}-\frac{1}{2} \int \frac{\rho\left(\vec{r}_{1}\right) \rho\left(\vec{r}_{2}\right)}{r_{12}} d \vec{r}_{1} d \vec{r}_{2}+E_{x c}[\rho]$

O desafio na DFT é encontrar a expressão exata para o termo de troca-correlação, $E_{x c}[\rho]$. Assim, existe uma grande diversidade de representações e aproximações para ele. Desde expressões locais que consideram as contribuições de troca e correlação separadamente, como os funcionais puro de troca (Slater, Becke, PW91X e PBEX) e de correlação (VWN, VWN5, B86 e LYP), bem como combinados (SVWN, BLYP, BP86, PW91 e PBE). Outras possibilidades são definidas em termos das aproximações generalizadas de gradiente (do inglês GGA) e aí aparecem também expressões que consideram contribuições da parte de troca utilizando-se o método HF (os funcionais híbridos). Um dos potenciais mais populares que representa esta categoria é o B3LYP (um funcional que combina 5 funcionais, Becke + Slater + troca HF + LYP e VWN5). Atualmente tem sido muito utilizados os funcionais denominados meta-GGA, entre estes a família M06 (funcional de troca-correlação híbrido com $27 \%$ de troca de HF), ${ }^{23}$ sendo M06-2X, o M06 com 54\% de troca HF, empregado neste trabalho.

Os métodos teóricos descritos são aplicáveis para o estado fundamental. Para o estudo em estados excitados, excetuando-se os métodos multiconfiguracionais, alternativas que têm sido bastante empregadas são aquelas baseadas na dependência temporal ou equações de movimento. A teoria do funcional de densidade dependente do tempo (do inglês TD-DFT) é uma extensão da DFT para problemas dependentes do tempo e sua base teórica é dada pelo teorema de Runge-Gross. ${ }^{24} \mathrm{Na}$ TD-DFT, o funcional passa a ser densidade eletrônica $\rho(\vec{r}, t)$. Como o tempo passa a ser variável da densidade, o estado fundamental é o caso particular para $t_{0}$. Assim, para um mesmo sistema a diferença da densidade eletrônica considerando-se tempos distintos leva a estados eletrônicos distintos.

Os métodos EOM-CC são baseados no formalismo do método das equações-de-movimento (do inglês EOM) para Coupled Cluster ${ }^{25,26} \mathrm{O}$ procedimento parte da representação de um estado excitado como uma excitação de um estado fundamental descrito pelo Coupled Cluster e as energias de excitação são obtidas resolvendo um problema de autovalor não-Hermitiano. A teoria de mais alto nível CR-EOM$\operatorname{CCSD}(\mathrm{T})$, utilizada neste trabalho, corresponde à representação do método de Coupled Cluster completamente renormalizado com excitações completas de simples e duplas e triplas não interativas baseados nas equações de movimento para CC.

\section{METODOLOGIA COMPUTACIONAL}

Este trabalho está dividido em duas partes. No primeiro estudo foram feitos cálculos teóricos das estruturas dos sistemas moleculares: etileno $\left(\mathrm{C}_{2} \mathrm{H}_{4}\right)$, ciclo butadieno $\left(\mathrm{C}_{4} \mathrm{H}_{4}\right)$, trans-1,3 butadieno $\left(\mathrm{C}_{4} \mathrm{H}_{6}\right)$, benzeno $\left(\mathrm{C}_{6} \mathrm{H}_{6}\right)$, trans-1,3,5 hexatrieno $\left(\mathrm{C}_{6} \mathrm{H}_{8}\right)$, "cubeno $\left(\mathrm{C}_{8}\right)$ " (neologismo em analogia ao sistema cubano $\left(\mathrm{C}_{8} \mathrm{H}_{8}\right)$ e por conter apenas ligações duplas), fulereno $\left(\mathrm{C}_{60}\right)$ e corantes de cianinas simétricas $\left(\left[\mathrm{H}_{2} \mathrm{NCHCH}(\mathrm{CHCH})_{\mathrm{k}} \mathrm{CHNH}_{2}\right]^{+}\right.$, para $k=0$ a 4$)$. As geometrias moleculares dos estados fundamental e excitado foram pré-otimizadas no nível de teoria M06-2X/6-311++G** e TD(Nstates=5, Root=1)M06-2X/6-311++G**, respectivamente. Fez-se a caracterização dos pontos estacionários e a análise das frequências vibracionais harmônicas dos estados fundamentais. Estes foram reotimizados no nível de teoria CCSD/6-311++G**. Já para os estados excitados considerou-se cálculos EOM-CCSD/6-311++G**. A família de funcionais de densidade de troca-correlação M06 tem sido bastante testada em estudos que incluem barreiras de energia, análises conformacionais e energias de dissociação. ${ }^{27}$ M06 e M06-2X também foram desenvolvidos (e testados) para cálculos de estados eletronicamente excitados. ${ }^{23}$ Baseando-se nestes testes, o uso do funcional M06-2X foi escolhido por ser o mais indicado no estudo de sistemas contendo átomos do grupo principal. Já o conjunto de funções de base de Pople 6-311++G** apresentou excelentes resultados no estudo de estruturas de estados excitados, utilizando-se o método EOM-CCSD. Para alguns sistemas menores $\left(\mathrm{C}_{2} \mathrm{H}_{4}, \mathrm{C}_{4} \mathrm{H}_{4}, \mathrm{C}_{4} \mathrm{H}_{6}\right.$ e $\left.\mathrm{C}_{6} \mathrm{H}_{6}\right)$ energias dos estados singletos excitados foram obtidas no nível de teoria $\mathrm{CR}$ EOM-CCSD(T) (método de equações de movimento completamente renormalizadas com excitações simples, duplas e triplas não interativas). ${ }^{28} \mathrm{Na}$ segunda parte, os cálculos dos comprimentos de onda referentes às transições eletrônicas entre os estados fundamental e 
1 excitado foram feitos segundo os modelos da partícula na caixa (1D, 2D ou 3D), no anel ou na superfície esférica.

Todos os cálculos de estrutura eletrônica foram feitos utilizando-se os programas Gaussian0929 ou Gamess/2010. ${ }^{30}$

\section{RESULTADOS E DISCUSSÃO}

\section{Métodos de estrutura eletrônica}

Na Tabela 1 estão as energias eletrônicas obtidas para os estados singleto fundamental - EF (S) e excitado - EE (S) calculadas nos níveis de teoria CCSD, EOM-CCSD e TD-M06-2X com conjuntos de base $6-311++\mathrm{G}^{* *}$. As energias dos estados excitados referem-se a processos de transições eletrônicas adiabática (AD) e vertical de Flanck-Condon (FC), efetuados neste último caso, na geometria otimizada do estado fundamental. Os resultados obtidos a partir dos cálculos mais precisos, CR-EOM-CCSD(T), melhoraram muito pouco os resultados das transições eletrônicas, e em alguns casos, como no benzeno, forneceram resultados piores, talvez pelo fato do cálculo ter sido feito na geometria do nível CCSD. Para os sistema maiores o custo computacional em cálculos no nível CR-EOM-CCSD(T) é proibitivo. No caso da molécula $\mathrm{C}_{60}$ não foi possível efetuar nenhum tipo de cálculo, com precisão razoável para reproduzir o espectro eletrônico, em virtude do tamanho do sistema.

Dados experimentais para o espectro eletrônico do ciclobutadieno

Tabela 1. Energias eletrônicas (em u.a.) dos estados singletos fundamental - EF $\left(\mathrm{S}_{0}\right)$ e excitado - $\mathrm{EE}\left(\mathrm{S}_{1}\right)$

\begin{tabular}{ccrrr}
\hline \multirow{2}{*}{$\begin{array}{c}\text { Sistema } \\
\text { molecular }\end{array}$} & $\begin{array}{c}\text { Grupo pon- } \\
\text { tual }\left(\mathrm{S}_{0}\right)\end{array}$ & $\mathrm{EF}\left(\mathrm{S}_{0}\right)$ & \multicolumn{2}{c}{$\mathrm{EE}\left(\mathrm{S}_{1}\right)^{(\mathrm{a})}$} \\
\cline { 4 - 5 } $\mathrm{CCSD}^{(\mathrm{b})}$ & & & & $\mathrm{AD}$ \\
\hline $\mathrm{C}_{2} \mathrm{H}_{4}$ & $\mathrm{D}_{2 \mathrm{~h}}$ & $-78,41685$ & $-78,19623$ & $-78,14507$ \\
$\mathrm{C}_{4} \mathrm{H}_{4}$ & $\mathrm{D}_{2 \mathrm{~h}}$ & $-154,35229$ & $-154,25699$ & $-154,22277$ \\
& $\mathrm{D}_{4 \mathrm{~h}}$ & $-154,31806$ & $-154,25699$ & $-154,25736$ \\
$\mathrm{C}_{4} \mathrm{H}_{6}$ & $\mathrm{C}_{2 \mathrm{~h}}$ & $-155,65473$ & $-155,42415$ & $-155,42065$ \\
$\mathrm{C}_{6} \mathrm{H}_{8}$ & $\mathrm{C}_{2 \mathrm{~h}}$ & $-232,76490$ & $-232,56684$ & $-232,58341$ \\
$\mathrm{C}_{6} \mathrm{H}_{6}$ & $\mathrm{D}_{6 \mathrm{~h}}$ & $-231,62576$ & $-231,43962$ & $-231,43476$ \\
$\mathrm{C}_{8}$ & $\mathrm{O}_{\mathrm{h}}$ & $-303,56675$ & $-303,47281$ & $-303,47122$ \\
$\mathrm{C}_{60}$ & $\mathrm{I}_{\mathrm{h}}$ & $\mathrm{nd} \mathrm{d}^{(\mathrm{c})}$ & $\mathrm{nd}$ & $\mathrm{nd}$ \\
\hline $\mathrm{CR}_{-} \mathrm{CCSD}(\mathrm{T})^{(\mathrm{b})}$ & & & & \\
\hline $\mathrm{C}_{2} \mathrm{H}_{4}$ & $\mathrm{D}_{2 \mathrm{~h}}$ & $-78,42789$ & $-78,21547$ & $-78,15276$ \\
$\mathrm{C}_{4} \mathrm{H}_{4}$ & $\mathrm{D}_{2 \mathrm{~h}}$ & $-154,37529$ & $-154,27222$ & $-154,23618$ \\
& $\mathrm{D}_{4 \mathrm{~h}}$ & $-154,34584$ & $-154,27222$ & $-154,27326$ \\
$\mathrm{C}_{4} \mathrm{H}_{6}$ & $\mathrm{C}_{2 \mathrm{~h}}$ & $-155,67541$ & $-155,43809$ & $-155,43055$ \\
$\mathrm{C}_{6} \mathrm{H}_{6}$ & $\mathrm{D}_{6 \mathrm{~h}}$ & $-231,65545$ & $-231,46502$ & $-231,45935$ \\
\hline $\mathrm{M}_{06}-2 \mathrm{X}^{(\mathrm{b})}$ & & & & \\
\hline $\mathrm{K}_{0}$ & $\mathrm{C}_{\mathrm{s}}$ & $-227,75878$ & $-227,57568$ & $-227,56742$ \\
$\mathrm{~K}_{1}$ & $\mathrm{C}_{\mathrm{s}}$ & $-305,14372$ & $-304,99713$ & $-304,99408$ \\
$\mathrm{~K}_{2}$ & $\mathrm{C}_{\mathrm{s}}$ & $-382,52628$ & $-382,40290$ & $-382,40131$ \\
$\mathrm{~K}_{3}$ & $\mathrm{C}_{\mathrm{s}}$ & $-459,90775$ & $-459,80078$ & $-459,79916$ \\
$\mathrm{~K}_{4}$ & $\mathrm{C}_{\mathrm{s}}$ & $-537,28848$ & $-537,19369$ & $-537,19230$ \\
\hline & & & & \\
\hline
\end{tabular}

(a) $\mathrm{AD}$ e FC correspondem às energias associadas aos processos de transições adiabática e vertical de Flanck-Condon, respectivamente. ${ }^{(b)}$ Cálculos dos estados fundamentais usando CCSD, CR-CCSD(T) ou M06-2X e excitados com EOM-CCSD, CR-EOM-CCSD $(\mathrm{T})$ ou TD $($ Nstates $=5$, Root $=1$ ) $/ \mathrm{M} 06-2 \mathrm{X}$ e conjuntos de funções de base 6-311++G**. ${ }^{(c)}$ Não disponível. são escassos e contraditórios. Medidas recentes do espectro de absorção no UV mostram somente um pico intenso por volta de $200 \mathrm{~nm}$ e uma fraca absorção que se estende até quase $500 \mathrm{~nm} .{ }^{31}$ É um sistema singular. O estado fundamental apresenta a conformação retangular $\left(D_{2 h}\right)$ como a forma mais estável em aproximadamente 0,2 u.a. se comparada com a forma quadrada de simetria $\mathrm{D}_{4 \mathrm{~h}}$. No entanto, o primeiro estado excitado tem geometria quadrada. ${ }^{32}$

A Tabela 2 apresenta os comprimentos de onda calculados para a transição eletrônica $\pi \rightarrow \pi^{*}$ nos estados de simetria de spin singleto e resultados experimentais correspondentes para os sistemas estudados. Os comprimentos de onda foram obtidos através das diferenças de energias $(\Delta E)$ entre dois níveis consecutivos, dados pela Equação 16. As energias consideradas são aquelas da Tabela 1. Observa-se que os resultados obtidos através dos cálculos no nível de teoria EOM-CCSD/6-311++G** estão em excelente acordo com os valores experimentais. Vale salientar que a "molécula $\mathrm{C}_{8}$ " é uma representação hipotética e puramente teórica do que seria um sistema conjugado em uma caixa tridimensional, e será discutida adiante.

Tabela 2. Comprimentos de onda (em nm) calculados para a transição eletrônica $\pi \rightarrow \pi^{*}$ na simetria de spin singleto e resultados experimentais

\begin{tabular}{ccccc}
\hline \multirow{2}{*}{$\begin{array}{c}\text { Sistema } \\
\text { molecular }\end{array}$} & \multirow{2}{*}{$\begin{array}{c}\text { Grupo } \\
\text { pontual }\end{array}$} & \multicolumn{3}{c}{$\lambda$} \\
\cline { 5 - 5 } CCSD $^{(a)}$ & & & FC & Exp. \\
\hline $\mathrm{C}_{2} \mathrm{H}_{4}$ & $\mathrm{D}_{2 \mathrm{~h}}$ & 206,5 & 167,5 & $163,1^{38}-225,4^{39}$ \\
$\mathrm{C}_{4} \mathrm{H}_{4}$ & $\mathrm{D}_{2 \mathrm{~h}}$ & 478,1 & 351,8 & $200,0-495,9^{31}$ \\
& $\mathrm{D}_{4 \mathrm{~h}}$ & 746,0 & 750,6 & - \\
$\mathrm{C}_{4} \mathrm{H}_{6}$ & $\mathrm{C}_{2 \mathrm{~h}}$ & 197,6 & 194,7 & $209,8^{40}$ \\
$\mathrm{C}_{6} \mathrm{H}_{8}$ & $\mathrm{C}_{2 \mathrm{~h}}$ & 251,0 & 230,0 & $241,7^{41}$ \\
$\mathrm{C}_{6} \mathrm{H}_{6}$ & $\mathrm{D}_{6 \mathrm{~h}}$ & 244,8 & 238,5 & $253,0^{42}$ \\
$\mathrm{C}_{8}$ & $\mathrm{O}_{\mathrm{h}}$ & 485,0 & 476,9 & $n d^{(\mathrm{b})}$ \\
$\mathrm{C}_{60}$ & $\mathrm{I}_{\mathrm{h}}$ & $\mathrm{nd}$ & $\mathrm{nd}$ & $407^{43}$ \\
\hline $\mathrm{M} 06-2 \mathrm{X}$ & & & & \\
\hline $\mathrm{K}_{0}$ & $\mathrm{C}_{\mathrm{s}}$ & 248,8 & 238,1 & $313^{44}$ \\
$\mathrm{~K}_{1}$ & $\mathrm{C}_{\mathrm{s}}$ & 310,8 & 304,5 & $416^{44}$ \\
$\mathrm{~K}_{2}$ & $\mathrm{C}_{\mathrm{s}}$ & 369,3 & 364,6 & $519^{44}$ \\
$\mathrm{~K}_{3}$ & $\mathrm{C}_{\mathrm{s}}$ & 425,9 & 419,6 & $625^{44}$ \\
$\mathrm{~K}_{4}$ & $\mathrm{C}_{\mathrm{s}}$ & 480,7 & 473,7 & $723^{37}$ \\
\hline
\end{tabular}

(a) Os valores para $\lambda$ para as transicões $\mathrm{AD} \mathrm{e}(\mathrm{FC})$ dos sistemas $\mathrm{C}_{2} \mathrm{H}_{4}, \mathrm{C}_{4} \mathrm{H}_{4}\left(\mathrm{D}_{2 \mathrm{~h}}\right)$, $\mathrm{C}_{4} \mathrm{H}_{4}\left(\mathrm{D}_{4 \mathrm{~h}}\right), \mathrm{C}_{4} \mathrm{H}_{6}$ e $\mathrm{C}_{6} \mathrm{H}_{6}$ calculados usando o nível de teoria CR-CCSD $(\mathrm{T}) / 6$ $311++\mathrm{G}^{* *} / / \mathrm{CCSD} / 6-311++\mathrm{G}^{* *}$ são 214,5 (172,5); 442,1 (392,5); 618,9 $(1017,1) ; 192,0(203,2)$ e 283,5 $(273,8)$ nm, respectivamente. ${ }^{(b)}$ Não disponível.

Os resultados calculados com os métodos EOM-CCSD, EOMCCSD(T) e conjuntos de base 6-311++G** apresentaram resultados muito próximos aos obtidos experimentalmente. Já no caso dos corantes de cianinas simétricas $\left(\left[\mathrm{H}_{2} \mathrm{~N} \mathrm{CHCH}(\mathrm{CHCH})_{\mathrm{k}} \mathrm{CHNH}_{2}\right]^{+}\right)$, os resultados obtidos com TD-DFT assemelham-se aos valores experimentais apenas para os sistemas menores. Os valores de $\lambda$ utilizando-se os modelos da partícula "livre" confinada estão na Tabela 3 e serão discutidos a seguir.

\section{Modelos da partícula "livre" confinada}

\section{$\mathrm{C}_{2} \mathrm{H}_{4}$ e dienos conjugados $\left(\mathrm{C}_{4} \mathrm{H}_{6}\right.$ e $\left.\mathrm{C}_{6} \mathrm{H}_{8}\right)$}

No caso do modelo da partícula na caixa unidimensional, as transições eletrônicas dos elétrons "livres" nos dienos conjugados se dão entre os orbitais de fronteira, $n_{\text {номо }}$ e $n_{\text {LUмO }}$. O valor de $n_{\text {номо }}$ é 
Tabela 3. Parâmetros geométricos (em $\AA$ ), $\mathrm{n}_{\text {номо }}$ (Highest Occupied Molecular Orbital), e comprimentos de onda (em nm) calculados e experimentais

\begin{tabular}{|c|c|c|c|c|c|c|c|c|}
\hline \multirow{2}{*}{$\begin{array}{l}\text { Sistema } \\
\text { molecular }\end{array}$} & \multirow[t]{2}{*}{ Modelo } & \multicolumn{3}{|c|}{ Dimensões } & \multirow{2}{*}{$\mathrm{n}_{\text {HOMO }}$} & \multicolumn{3}{|c|}{$\lambda$} \\
\hline & & $a$ & $b$ & $c$ & & Mod. & Calc. & Exp. \\
\hline $\mathrm{C}_{2} \mathrm{H}_{4}$ & Cx 1D & 2,87 & - & - & 1 & 90,5 & 206,5 & $163,1-225,4$ \\
\hline $\mathrm{C}_{4} \mathrm{H}_{4}$ & Cx 1D & 5,68 & - & - & 2 & 212,8 & 197,6 & 209,8 \\
\hline $\mathrm{C}_{4} \mathrm{H}_{6}$ & Cx 1D & 8,48 & - & - & 3 & 338,7 & 230,0 & 241,7 \\
\hline $\mathrm{C}_{6} \mathrm{H}_{8}$ & $\mathrm{Cx} 2 \mathrm{D}$ & 2,43 & 2,67 & - & 1 & 285,9 & 478,1 & $200,0-495,9$ \\
\hline \multirow[t]{2}{*}{$\mathrm{C}_{6} \mathrm{H}_{6}$} & Cx 2D & 4,35 & 4,35 & - & 3 & 263,9 & 244,8 & 253,0 \\
\hline & Anel $^{(\mathrm{b})}$ & - & & - & 3 & 225,0 & - & - \\
\hline \multirow[t]{2}{*}{$\mathrm{C}_{8}$} & Cx 3D & 1,47 & 1,47 & 1,47 & 4 & 23,8 & 485,0 & $\mathrm{nd}^{(\mathrm{d})}$ \\
\hline & Cx 1D & 8,82 & - & - & 4 & 366,4 & - & - \\
\hline \multirow[t]{2}{*}{$\mathrm{C}_{60}$} & Cx 3D & 7,00 & 7,00 & 7,00 & 16 & - & nd & 407 \\
\hline & Esfera $^{(\mathrm{c})}$ & - & - & - & 16 & 404 & - & - \\
\hline $\mathrm{K}_{0}$ & Cx 1D & 7,18 & - & - & 3 & 242,8 & 248,8 & 313 \\
\hline $\mathrm{K}_{1}$ & Cx 1D & 9,97 & - & - & 4 & 364,5 & 310,8 & 416 \\
\hline $\mathrm{K}_{2}$ & Cx 1D & 12,77 & - & - & 5 & 488,5 & 369,3 & 519 \\
\hline $\mathrm{K}_{3}$ & Cx 1D & 15,55 & - & - & 6 & 613,0 & 425,9 & 625 \\
\hline $\mathrm{K}_{4}$ & Cx 1D & 18,35 & - & - & 7 & 740,3 & 480,7 & 723 \\
\hline
\end{tabular}

${ }^{(\mathrm{a})} n_{\text {HOMO }}=\frac{N^{o} \bar{e} \pi}{2} \cdot{ }^{(\mathrm{b})} r_{\text {Anel }}=2,20 \AA .{ }^{(\mathrm{c})} r_{\text {Esfera }}=6,50 \AA .{ }^{(\mathrm{d})}$ Não disponível.

determinado pela metade do número de elétrons $\pi$. Embora o etileno não seja um dieno conjugado, ele será considerado neste grupo. Assim, o comprimento de onda máximo associado à diferença de energias entre os estados com $n_{\text {номо }}$ e $n_{\text {LUMO }}$, que reproduz bem os resultados experimentais, corrigindo entre outras aproximações os efeitos de borda da cadeia carbônica e o potencial nulo, através do ajuste do comprimento da caixa, é dado substituindo-se apropriadamente os valores na Equação $16,{ }^{33}$ por

$$
\lambda_{\max }=\frac{329,7}{2 n_{\text {HOMO }}+1} r^{2}
$$

onde $2 n_{\text {номо }}+1=n_{\text {LUмо }}{ }^{2}-n_{\text {номо }}{ }^{2}$ e $r$ o comprimento da caixa. Para a molécula $\mathrm{C}_{2} \mathrm{H}_{4}$ a distância de deslocamento do elétron pode ser estimada através da soma dos comprimentos das ligação $\mathrm{C}=\mathrm{C}(1,33 \AA)$ mais a distância de um raio do átomo de carbono de cada extremidade da cadeia ( 2 x $0,77 \AA$ ), totalizando-se $2,87 \AA$. No caso de $\mathrm{C}_{4} \mathrm{H}_{6}$ o comprimento da caixa é igual a 2 vezes o comprimento das ligações $\mathrm{C}=\mathrm{C}(2 \times 1,34 \AA)$ mais um comprimento de uma ligação simples C-C $(1,46 \AA)$ e 2 x $0,77 \AA$, dando uma distância total de 5,68 ̊. Para o $1,3,5$ hexatrieno $\left(\mathrm{C}_{4} \mathrm{H}_{8}\right)$ o valor é $3 \times 1,34+2 \times 1,46+2 \times 0,77$ e igual a $8,48 \AA$ A. Os valores de $\lambda_{\text {max }}$ calculados, como indicado na Tabela 3 para este três sistemas são 90,5; 212,8 e 338,7 nm, respectivamente.

O modelo da partícula na caixa é um modelo bastante simples que não considera qualquer efeito da ação do potencial, quer de natureza atrativa ou repulsiva. Assim, é de se esperar que sua validade também seja limitada. Para se ter uma reprodutibilidade em relação a dados experimentais ou de cálculos (rigorosos) de estrutura eletrônica faz-se necessário ajustes em um conjunto de sistemas moleculares, como o procedimento descrito acima. Não há garantia que a expressão obtida a partir destes ajustes seja também adequada em outros sistemas não presentes neste conjunto como, por exemplo, em sistemas com um maior número de conjugações.

Mesmo entre os métodos de cálculos teóricos há discrepâncias entre os resultados para absorção e/ou emissão a partir das diferenças de energias entre estados eletrônicos. Por exemplo, o uso de estimativas de energias orbitais em cálculos do tipo Hartree-Fock é severamente desaconselhado neste tipo de estudo. Embora a contribuição da correlação eletrônica na energia, negligenciada pelo HF, seja relativamente pequena, seus efeitos nas diferenças de energias envolvidas nas transições eletrônicas são bastantes significativos. A inclusão de correlação eletrônica também não é garantia da qualidade do resultado. No caso da TD-DFT, por exemplo, a escolha adequada do funcional de troca-correlação é relevante. Para a teoria EOM-CC, fatores como renormalização e/ou nível de excitação influenciam na qualidade do resultado. E em ambas as teorias, a escolha do conjunto de funções de base também afetará os valores associados às transições eletrônicas.

\section{$\mathrm{C}_{4} \mathrm{H}_{4}, \mathrm{C}_{6} \mathrm{H}_{6}, \mathrm{C}_{8}$ e $\mathrm{C}_{60}$}

As moléculas de $\mathrm{C}_{4} \mathrm{H}_{4}$ e $\mathrm{C}_{6} \mathrm{H}_{6}$ podem ser representadas por um modelo de partícula na caixa em 2D. Para isto deve-se inscrevê-las em caixas retangular e quadrada, de dimensões 2,43 x 2,67 e 4,35 x $4,35 \AA$, respectivamente. Estes valores de comprimentos das caixas foram obtidos considerando-se a adição da distância de um raio do átomo de carbono $(0,77 \AA)$. $\mathrm{Em}_{4} \mathrm{H}_{4}$ os 4 elétrons $\pi$ ocupam os níveis de energia $\mathrm{E}_{1,1}$ e $\mathrm{E}_{2,1}$ ou $\mathrm{E}_{1,2}$ e a transição ocorre entre os níveis $\mathrm{E}_{1,2}$ ou $\mathrm{E}_{2,1}$ para $\mathrm{E}_{2,2}$. O comprimento de onda associado a esta transição é 287,0 nm. Já para a molécula de benzeno, os 6 elétrons $\pi$ possuem energias iguais a $E_{1,1}, E_{1,2}$ e $E_{2,1}$. A transição de menor energia acontece de $E_{1,2}$ ou $E_{2,1}$ para $E_{2,2}$. Como o benzeno inscrito em uma caixa bidimensional possui dimensões $a=4,35 \AA$, o valor obtido para o comprimento de onda é $207,0 \mathrm{~nm}$. Se a representação para o benzeno se der em termos de um anel de raio $r$, definido como sendo a medida entre o centro do anel mais o fator de borda de 0,77 Åe igual a 2,20 Å(Figura 1a) o valor de $\lambda$ é $225,0 \mathrm{~nm}$. O valor ótimo de $r$ para reproduzir o dado experimental deveria ser $2,32 \AA$.

A representação do $\mathrm{C}_{60}$ por uma superfície esférica é mais realista do que a molécula inscrita numa caixa cúbica, embora de fato, sua estrutura seja reconhecida como um icosaedro truncado. A molécula de $\mathrm{C}_{60}$ contém 12 pentágonos e 20 hexágonos (Figura $1 \mathrm{~b}$ ). As ligações entre os 60 átomos de carbono situados nos vértices desta estrutura formam 90 ligações covalentes, sendo 60 ligações simples e 30 ligações $\pi$. No entanto, nem todas as duplas ligações possuem a mesma energia, pois há átomos localizados em diferentes tipos de vértices. Considerando-se o $\mathrm{C}_{60}$ como sendo uma esfera perfeita de raio igual a $6,5 \AA$ (obtido a partir de sua geometria experimental) e ajustando-se o valor de $n_{\text {номо }}$ para reproduzir o valor de $\lambda_{\text {exp }}$, obtém-se para este um valor de 16. No outro extremo, para $n_{\text {номо }}=30$, o valor de $\lambda$ é $221,8 \mathrm{~nm}$. Se utilizarmos os valores de $n_{\text {номо }}$ entre os 
limites 16 e 30 na caixa 3D os valores de $\lambda$ obtidos são iguais a 48,9 e $26,5 \mathrm{~nm}$, respectivamente, muito distantes do valor experimental.

Um modelo adequado para partícula numa caixa 3D, seria o uso da molécula hipotética de $\mathrm{C}_{8},{ }^{34}$ a qual poderia ser obtida através da desidrogenação do hidrocarboneto pentaciclo[4.2.0.02,5.03,8.04,7] octano, $\mathrm{C}_{8} \mathrm{H}_{8}$ (cubano), como sugerido pela seguinte reação:

$$
\mathrm{C}_{8} \mathrm{H}_{8} \rightarrow \mathrm{C}_{8}+4 \mathrm{H}_{2}
$$

O confinamento da partícula no cubo serve para quantizar sua energia e momento:

$$
E=\frac{1}{2 m}\left(p_{x}^{2}+p_{y}^{2}+p_{z}^{2}\right)
$$

sendo necessários três números quânticos para especificar a condição quântica correspondente aos três graus de liberdade independentes para a partícula no espaço. $\mathrm{O}$ estado fundamental, $\operatorname{com} n_{1}=n_{2}=n_{3}$ $=1$, tem energia

$$
E_{111}=\frac{3 h^{2}}{8 m_{e} r^{2}}
$$

Existem três primeiros estados excitados que correspondem a três combinações diferentes de $n_{1}, n_{2}$ e $n_{3}$. Estas três combinações dão a energia do primeiro estado excitado, triplamente degenerada e igual a

$$
E_{211}=E_{121}=E_{112}=\frac{6 h^{2}}{8 m_{e} r^{2}}
$$

Este sistema tem níveis degenerados por causa do alto grau de simetria associado à forma cúbica da caixa. O segundo estado excitado corresponde a três combinações dos números quânticos $n_{1}=2, n_{2}=2$ e $n_{3}=1$ ou $n_{1}=2, n_{2}=1$ e $n_{3}=2$ ou $n_{1}=1, n_{2}=2$ e $n_{3}=2$ e novamente os níveis de energia são triplamente degenerados

$$
E_{221}=E_{212}=E_{122}=\frac{9 h^{2}}{8 m_{e} r^{2}}
$$

Observou-se que o modelo acima não é satisfatório para a estimativa do comprimento de onda para o $\mathrm{C}_{8}$. Para a caixa cúbica de comprimento $r=1,47 \AA$, as transições entre o estado fundamental e o primeiro estado excitado, ou entre este último estado e o segundo estado excitado, são iguais a $23,75 \mathrm{~nm}$, ou seja, muito distantes do valor de 480,7 nm estimado por cálculo teórico. Uma sugestão de abordagem, que melhorou significativamente o resultado, foi considerar o delocalização eletrônica na caixa como um todo, como mostra a Figura 2. O caminho percorrido por uma dada partícula corresponde a 6 vezes o comprimento da caixa $1 \mathrm{D}(8,82 \AA)$. Os valores de $\lambda$ obtidos para as transições entre níveis consecutivos do estado fundamental até $n_{50}$ são mostrados na Figura 3a. Observa-se o caráter convergente de $\lambda$ x $n$ e em $n$ entre 3 e $4\left(n_{\text {номо }}=4\right)$, resultado próximo ao valor estimado pelo cálculo EOM-CCSD/6-311++G**

\section{Corantes de cianinas}

É bastante comum o uso de modelos da partícula na caixa em disciplinas de Físico-Química experimental, pois integram muito bem conceitos de química quântica com espectroscopia no visível. ${ }^{35,36}$ Para isto a delocalização eletrônica é explicada considerando-se estruturas de corantes, melhor que polienos conjugados, por apresentarem os primeiros absorção na região do visível do espectro eletromagnético. ${ }^{37}$ Assim, escolhemos uma classe de corantes de cianinas simétricas, $\mathrm{HN}_{2} \mathrm{CH}=\mathrm{CH}(\mathrm{CH}=\mathrm{CH})_{\mathrm{k}} \mathrm{CH}=\mathrm{NH}_{2}{ }^{+}$para $k=0,1$,

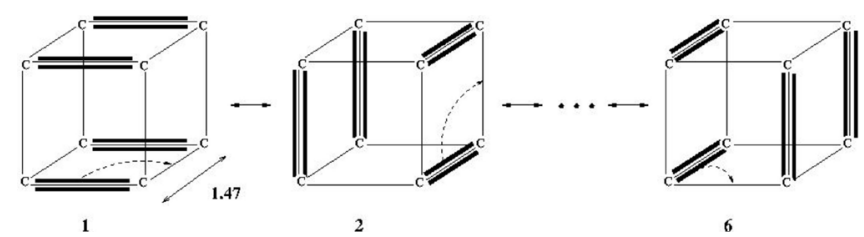

Figura 2. Delocalização eletrônica para a molécula hipotética " $C_{8}$ "

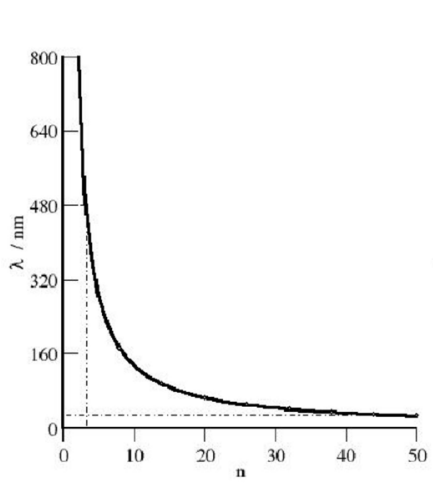

(a)

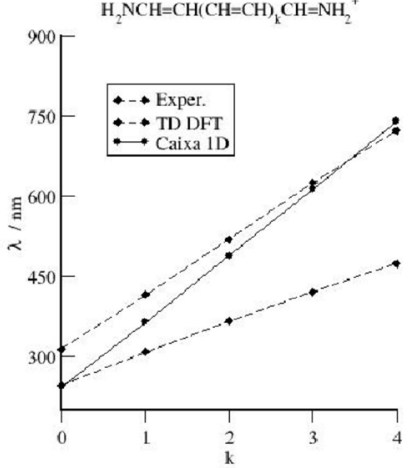

(b)
Figura 3. Comprimento de onda (em nm) em função de: (a) n para as transições eletrônicas em " $C_{8}$ " $e(b) k$, para o corante de cianinas simétricas $\mathrm{H}_{2} \mathrm{NCH}=\mathrm{CH}(\mathrm{CH}=\mathrm{CH})_{k} \mathrm{CH}=\mathrm{NH}_{2}{ }^{+}, \mathrm{k}=\mathrm{O} a 4$

2, 3 ou 4, onde o modelo da partícula na caixa em 1D se comporta bem. $\mathrm{O}$ ajuste aos dados experimentais é obtido considerando-se a expressão dada pela Equação 19. No entanto, o valor de $r$ é calculado considerando-se os comprimentos das ligações NC e CC, obtidas da otimização da geometria molecular. O efeito de borda, graças às influência de grupos polarizáveis $\left(R R^{\prime} N-\right)$ no final da cadeia do corante, ${ }^{37}$ é compensado pela adição do raio atômico do átomo de $\mathrm{N}$ $(0,88 \AA)$. Na Figura 3 b observa-se uma melhor concordância entre os valores obtidos pelo modelo da partícula na caixa unidimensional e os de dados experimentais, principalmente com o aumento da cadeia carbônica, diferentemente daqueles calculados com o método TD-M06-2X/6-311++G**.

Há outros exercícios interessantes que podem ser explorados utilizando-se modelos de caixas 2D ou 3D como estruturas de grafeno (formas quadradas ou retangulares), nanotubo de carbono (na forma cilíndrica inscrita numa caixa 3D) ou outras que a imaginação permitir.

\section{CONCLUSÃO}

O modelo da partícula "livre" confinada, quer em uma caixa uni, bi ou tridimensional, em um anel ou em uma superfície esférica não reproduz exatamente resultados experimentais ou de cálculos teóricos sofisticados de espectros eletrônicos, no entanto, fornece tendências e mesmo em alguns casos valores de mesma ordem de grandeza. Mais que reproduzir valores de transições eletrônicas, o modelo permite de modo simples a apresentação e fixação de conceitos relevantes de química quântica, como quantização de níveis de energia, separação de variáveis, transições eletrônicas, degenerescência, efeito JahnTeller, entre outros.

Observamos neste trabalho, que o modelo da partícula, quando ajustado apropriadamente às condições de contorno e de confinamento em alguns sistemas $\left(\mathrm{C}_{2} \mathrm{H}_{4}, \mathrm{C}_{4} \mathrm{H}_{4}, \mathrm{C}_{4} \mathrm{H}_{6}, \mathrm{C}_{6} \mathrm{H}_{6}, \mathrm{C}_{6} \mathrm{H}_{8}, " \mathrm{C}_{8}\right.$ ", $\mathrm{C}_{60} \mathrm{e}$ $\left[\mathrm{H}_{2} \mathrm{NCHCH}(\mathrm{CHCH})_{k} \mathrm{CHNH}_{2}\right]^{+}$, onde $k$ varia de $\left.0 \mathrm{a} 4\right)$, permite a obtenção de resultados próximos aos de cálculos teóricos sofisticados, como os métodos EOM-CCSD/6-311++G** ou TD-M06-2X/6-311++G**. 


\section{AGRADECIMENTOS}

À infraestrutura do Instituto de Química da UNICAMP, ao apoio financeiro do Conselho Nacional de Desenvolvimento Científico (CNPq) e ao GridUNESP pelas facilidades computacionais.

\section{REFERÊNCIAS}

1. Peixoto, E. M. A.; Quim. Nova 1978, 1, 21.

2. Ferreira, M. M. C.; Porto, M. E. G.; Quim. Nova 1993, 16, 589.

3. Novak, I.; J. Chem. Educ. 2001, 78, 395.

4. Morgon, N. H.; Quim. Nova 2008, 31, 1869.

5. Miller, G. R.; J. Chem. Educ. 1979, 56, 709.

6. Luana, V.; Otero-de la Roza, A.; Blanco, M. A.; Recio, J. M.; Eur. J. Phys. 2010, 31, 101 .

7. Castanho, M. A. R. B.; J. Chem. Educ. 2002, 79, 1092.

8. Liang, Y. Q.; Zhang, H.; Dardenne, Y. X.; J. Chem. Educ. 1995, 72, 148.

9. Zhukov, A. A.; Finkelstein, G.; Jetp Lett. 2009, 89, 212.

10. Kolesov, B.; Phys. Chem. Miner. 2008, 35, 271.

11. Santos, A. C. F.; Rev. Bras. Ensino Fis. 2010, 32, 2303.

12. Jiang, H.; Baranger, H. H.; Yang, W. T.; Phys. Rev. B 2003, 68, 165337.

13. Ekimov, A. I.; Onushchenko, A.; Jetp Lett. 1981, 34, 345.

14. Fedichkin, L.; Yanchenko, M.; Valiev, K. A.; Nanotechnology 2000, 11 , 387.

15. Ma, X.; Xu, F.; Benavides, J.; Cloutier, S. G.; Org. Electron. 2012, 13, 525 .

16. McArthur, E. A.; Godbe, J. M.; Tice, D. B.; Weiss, E. A.; J. Phys. Chem C 2012, 116, 6136.

17. Rochefort, A.; Salahub, D. R.; Avouris, P.; J. Phys. Chem. B 1999, 103, 641.

18. Chandra, A. K.; Introductory Quantum Chemistry, Tata McGraw Hill Publishing Company: USA, 1994.

19. Morgon, N. H.; Custodio, R.; Quim. Nova 1995, 18, 44.

20. Duarte, H. A.; Quim. Nova 2001, 24, 501.

21. Hohenberg, P.; Kohn, W.; Phys. Rev. B 1964, 136, B864.

22. Kohn, W.; Sham, L. J.; Phys. Rev. 1965, 140, 1133.

23. Zhao, Y.; Truhlar, D.; Theor. Chem. Acc. 2008, 120, 215.

24. Runge, E.; Gross, E. K. U.; Phys. Rev. Lett. 1984, 52, 997.

25. Geertsen, J.; Rittby, M.; Bartlett, R.; Chem. Phys. Lett. 1989, 164, 57.

26. Kowalski, K.; Piecuch, P.; J. Chem. Phys. 2004, 120, 1715.

27. Zhao, Y.; Truhlar, D. G.; Chem. Phys. Lett. 2011, 502, 1.
28. Wloch, M.; Gour, J. R.; Kowalski, K.; Piecuch, P.; J. Chem. Phys. 2005, 122, 214107.

29. Frisch, M. J.; Trucks, G. W.; Schlegel, H. B.; Scuseria, G. E.; Robb, M. A.; Cheeseman, J. R.; Scalmani, G.; Barone, V.; Mennucci, B.; Petersson, G. A.; Nakatsuji, H.; Caricato, M.; Li, X.; Hratchian, H. P.; Izmaylov, A. F.; Bloino, J.; Zheng, G.; Sonnenberg, J. L.; Hada, M.; Ehara, M.; Toyota, K.; Fukuda, R.; Hasegawa, J.; Ishida, M.; Nakajima, T.; Honda, Y.; Kitao, O.; Nakai, H.; Vreven, T.; Montgomery, Jr., J. A.; Peralta, J. E.; Ogliaro, F.; Bearpark, M.; Heyd, J. J.; Brothers, E.; Kudin, K. N.; Staroverov, V. N.; Kobayashi, R.; Normand, J.; Raghavachari, K.; Rendell, A.; Burant, J. C.; Iyengar, S. S.; Tomasi, J.; Cossi, M.; Rega, N.; Millam, J. M.; Klene, M.; Knox, J. E.; Cross, J. B.; Bakken, V.; Adamo, C.; Jaramillo, J.; Gomperts, R.; Stratmann, R. E.; Yazyev, O.; Austin, A. J.; Cammi, R.; Pomelli, C.; Ochterski, J. W.; Martin, R. L.; Morokuma, K.; Zakrzewski, V. G.; Voth, G. A.; Salvador, P.; Dannenberg, J. J.; Dapprich, S.; Daniels, A. D.; Farkas, Ö.; Foresman, J. B.; Ortiz, J. V.; Cioslowski, J.; Fox, D. J.; Gaussian 09 Revision A.1, Gaussian Inc. Wallingford CT, 2009.

30. Schmidt, M. W.; Baldridge, K. K.; Boatz, J. A.; Elbert, S. T.; Gordon, M. S.; Jensen, J. H.; Koseki, S.; Matsunaga, N.; Nguyen, K. A.; Su, S. J.; Windus, T. L.; Dupuis, M.; Montgomery Jr., J. A.; J. Comput. Chem. 1993, 14, 1347.

31. Arnold, B. R.; Michl, J.; J. Phys. Chem. 1993, 97, 13348.

32. Levchenko, S. V.; Krylov, A. I.; J. Chem. Phys. 2004, 120, 175.

33. Braga, J. P.; Fundamentos de Química Quântica, Editora UFV: Viçosa, 2007.

34. Um trabalho mais completo a respeito das estruturas eletrônica e molecular desta molécula foi submetido para publicação.

35. Shalhoub, G. M.; J. Chem. Educ. 1997, 74, 1317.

36. Soltzberg, L. J.; J. Chem. Educ. 2001, 78, 1432.

37. Autschbach, J.; J. Chem. Educ. 2007, 84, 1840.

38. Robin, M. B.; Higher excited states of polyatomic molecules, Academic Press: New York, 1985, vol. 2.

39. Mcmurchie, L. E.; Davidson, E. R.; J. Chem. Phys. 1977, 67, 5613.

40. McDiarmid, R.; Advances in Chemical Physics, John Wiley \& Sons Inc.: New York, 1999, vol. 110.

41. Kuppermann, A.; Flicker, W. M.; Mosher, O. A.; Chem. Rev. 1979, 79, 77.

42. Li, X.; Paldus, J.; J. Chem. Phys. 2011, 134, 214118.

43. Orlandi, G.; Negri, F.; Photochem. Photobiol. Sci. 2002, 1, 289.

44. Klessinger, M.; Chem. Unserer Zeit 1978, 12, 1. 